\title{
On Parameters Estimation of Lomax Distribution under General Progressive Censoring
}

\author{
Bander Al-Zahrani ${ }^{1}$ and Mashail Al-Sobhi ${ }^{2}$ \\ ${ }^{1}$ Department of Statistics, Faculty of Sciences, King Abdulaziz University, Jeddah 21589, Saudi Arabia \\ ${ }^{2}$ Department of Mathematics, Umm Al-Qura University, Makkah, Saudi Arabia
}

Correspondence should be addressed to Bander Al-Zahrani; bmalzahrani@kau.edu.sa

Received 3 December 2012; Revised 19 February 2013; Accepted 9 March 2013

Academic Editor: Antoine Grall

Copyright (C) 2013 B. Al-Zahrani and M. Al-Sobhi. This is an open access article distributed under the Creative Commons Attribution License, which permits unrestricted use, distribution, and reproduction in any medium, provided the original work is properly cited.

We consider the estimation problem of the probability $S=P(Y<X)$ for Lomax distribution based on general progressive censored data. The maximum likelihood estimator and Bayes estimators are obtained using the symmetric and asymmetric balanced loss functions. The Markov chain Monte Carlo (MCMC) methods are used to accomplish some complex calculations. Comparisons are made between Bayesian and maximum likelihood estimators via Monte Carlo simulation study.

\section{Introduction}

The Lomax distribution, also called "Pareto type II" distribution is a particular case of the generalized Pareto distribution (GPD). The Lomax distribution has been used in the literature in a number of ways. For example, it has been extensively used for reliability modelling and life testing; see, for example, Balkema and de Haan [1]. It also has been used as an alternative to the exponential distribution when the data are heavy tailed; see Bryson [2]. Ahsanullah [3] studied the record values of Lomax distribution. Balakrishnan and Ahsanullah [4] introduced some recurrence relations between the moments of record values from Lomax distribution. The order statistics from nonidentical right-truncated Lomax random variables have been studied by Childs et al. [5]. Also, the Lomax model has been studied, from a Bayesian point of view, by many authors; see, for example, Arnold et al. [6] and El-Din et al. [7]. Howlader and Hossain [8] presented Bayesian estimation of the survival function of the Lomax distribution. Ghitany et al. [9] considered Marshall-Olkin approach and extended Lomax distribution. Cramer and Schmiedt [10] considered progressively type-II censored competing risks data from Lomax distribution. The Lomax distribution has applications in economics, actuarial modelling, queuing problems and biological sciences; for details, we refer to Johnson et al. [11].
A positive random variable $X$ is said to have the Lomax distribution, abbreviated as $X \sim L(\alpha, \beta)$, if it has the probability density function (pdf)

$$
f(x ; \alpha, \beta)=\alpha \beta(1+\beta x)^{-(\alpha+1)}, \quad x>0, \alpha, \beta>0 .
$$

Here, $\alpha$ and $\beta$ are the shape and the scale parameters, respectively. The survival function (sf) associated with (1) is

$$
\bar{F}(x ; \alpha, \beta)=(1+\beta x)^{-\alpha}, \quad x>0 .
$$

Further probabilistic properties of this distribution are given, for example, in Arnold [12].

This paper is concerned with the problem of estimating $S=P(Y<X)$ for Lomax based on general progressive censored data. The reliability of a component during a given period of time is defined as the probability that its strength $X$ exceeds the stress $Y$, and symbolically we write $S=P(Y<X)$. We assume $X$ and $Y$ to be independent, and each follows a Lomax distribution. A good overview on estimating $S$ can be found in the monograph of Kotz et al. [13]. Later, the problem of estimating $S$ attracted the attention of many authors; for example, see Baklizi [14], Raqab et al. [15], Kundu and Raqab [16], and Panahi and Asadi [17], and references cited therein.

The rest of the paper is organized as follows. In Section 2, we give a brief overview of the general progressive censoring. 
The maximum likelihood estimators (MLEs) are obtained in Section 3. In Section 4, we obtain Bayes estimators using the symmetric and asymmetric balanced loss functions. In Section 5, the MCMC methods are used to accomplish some complex calculations, and, therefore, comparisons are made between Bayesian and maximum likelihood estimators via Monte Carlo simulation study.

\section{General Progressive Censoring}

We refer to the paper of Soliman et al. [18, page 452], for introducing the general progressive censoring as follows. Consider a general type-II progressive censoring scheme, proposed by Balakrishnan and Sandhu [19]. This scheme of censoring can be explained as follows: at time $X_{0} \equiv 0, n$ randomly selected components were placed on a life test. The failure times of the first $r$ components to fail, $X_{1}, \ldots, X_{r}$, were not observed. At the time of the $(r+1)$ th failure, $X_{r+1: n}, R_{r+1}$ number of surviving components are removed from the test randomly and so on; at the time of the $(r+i)$ th observed failure, $X_{r+i: n}$, $R_{r+i}$ number of surviving components are removed from the test randomly; and finally, at the time of the $m$ th failure, the remaining $R_{m}=n-m-R_{r+1}-R_{r+2}-\cdots-R_{m-1}$ are removed from the test. Suppose that $X_{r+1: m: n} \leq X_{r+2: m: n} \leq$ $\cdots \leq X_{m: m: n}$ are the lifetimes of the completely observed components to fail and that $R_{r+1}, R_{r+2}, \ldots, R_{m}$ are the number of components removed from the test at these failure times, respectively. The $R_{i}$ 's, $m$, and $r$ are prespecified integers such that $0 \leq r<m \leq n, 0 \leq R_{i} \leq n-i$ for $i=r+1, \ldots, m-1$, and $R_{m}=n-m-\sum_{i=r+1}^{m-1} R_{i}$. The resulting $(m-r)$ ordered values $X_{r+1: m: n}, X_{r+2: m: n}, \ldots, X_{m: m: n}$ are appropriately referred to as general progressively type-II censored order statistics.

Also referring to Soliman et al. [18], it should be noted that (i) if $R_{i}=0$, for $i=r+1, \ldots, m-1$, and $R_{m}=n-m$, the general progressively type-II censoring scheme is reduced to the case of type-II doubly censored sample. (ii) If $r=0$, this scheme is reduced to the progressive type-II right censoring. (iii) If $r=0$ and $R_{i}=0$, for $i=r+1, \ldots, m-1$ so that $R_{m}=n-m$, the general progressively type-II censoring scheme is reduced to conventional type-II one-stage right censoring, where just the first $m$ usual order statistics are observed. (iv) If $r=0$ and $R_{i}=0$, for $i=r+1, \ldots, m$, so that $m=n$, the general progressively type-II censoring scheme is reduced to the case of no censoring (complete sample case), where all $n$ usual order statistics are observed. In this scheme $R_{1}, R_{2}, \ldots, R_{m}$ are all prefixed. Saraoglu et al. [20] discussed two examples showing the motivation behind the developments of the stress-strength models under censored samples. For more details, see Balakrishnan and Aggarwala [21].

Suppose that $n$ randomly selected components from the $\mathrm{L}(\alpha, \beta)$ are put in test. Further, let $X_{r+1: m: n}, X_{r+2: m: n}, \ldots$, $X_{m: m: n}$ denote a general progressively type-II censored sample from that population, with $\left(R_{r+1}, R_{r+2}, \ldots, R_{m}\right)$ being the progressive censoring scheme. For simplicity of notation, we will use $x_{i}$ instead of $x_{i: m: n}$, and then $\mathbf{x}=\left(x_{r+1}, \ldots, x_{m}\right)$ is the observed general progressive censored sample. The likelihood function for the parameters $\alpha$ and $\beta$ is then

$$
\mathscr{L}(\alpha, \beta \mid \mathbf{x})=c^{*}\left\{1-\bar{F}\left(x_{r+1}\right)\right\}^{r} \prod_{i=r+1}^{m} f\left(x_{i}\right)\left[\bar{F}\left(x_{i}\right)\right]^{R_{i}},
$$

where

$$
c^{*}=\left(\begin{array}{l}
n \\
r
\end{array}\right)(n-r) \prod_{j=r+2}^{m}\left[n-\sum_{i=r+1}^{j-1} R_{i}-j+1\right],
$$

and the functions $f(x)$ and $\bar{F}(x)$ are given, respectively, by (1) and (2). Substituting (1) and (2) into (3), the likelihood function is

$$
\begin{aligned}
\mathscr{L}(\alpha, \beta \mid \mathbf{x})= & c^{*}\left[1-\left(1+\beta x_{r+1}\right)^{-\alpha}\right]^{r}(\alpha \beta)^{m-r} \\
& \times \prod_{i=r+1}^{m}\left(1+\beta x_{i}\right)^{-\alpha\left(1+R_{i}\right)-1} .
\end{aligned}
$$

Using the binomial expansion, $r$ is a positive integer, one can rewrite the likelihood function as follows:

$$
\mathscr{L}(\alpha, \beta \mid \mathbf{x})=c^{*}(\alpha \beta)^{m-r} e^{-u} \sum_{s=0}^{r} C_{r, s} e^{-\alpha T_{s}},
$$

where

$$
\begin{gathered}
u=u(\mathbf{x} ; \beta)=\sum_{i=r+1}^{m} \ln \left(1+\beta x_{i}\right), \\
T_{s}=T_{s}(\mathbf{x} ; \beta)=s \ln \left(1+\beta x_{r+1}\right) \\
-\sum_{i=r+1}^{m}\left(1+R_{i}\right) \ln \left(1+\beta x_{i}\right), \\
C_{r, s}=\left(\begin{array}{l}
r \\
s
\end{array}\right)(-1)^{s} .
\end{gathered}
$$

We focus our attention on the estimation of the probability $S=P(Y<X)$, where $X$ and $Y$ are two independent random variables each is $L\left(\alpha_{j}, \beta_{j}\right), j=1,2$ distributed, and the data obtained from both distributions are general progressively type-II censored. Here, $X$ and $Y$ are typically modeled as independent. The probability $P(Y<X)$ has been widely studied under different approaches and distributional assumptions on $X$ and $Y$. The case where $X$ and $Y$ are dependent has been considered by Nandi and Aich [22], Barbiero [23], and Rubio and Steel [24]. We investigate properties of $S$ when the common scale parameter $\beta_{1}=\beta_{2}=\beta$ is known. Then, it can be shown that

$$
S=P(Y<X)=\iint_{0<y<x} f(x, y) d x d y=\frac{\alpha_{1}}{\alpha_{1}+\alpha_{2}} .
$$

Here, $f(x, y)$ is the joint pdf of $X$ and $Y, f(x, y)=f_{1}(x) f_{2}(y)$ by the independence. The general case, when $\beta_{1} \neq \beta_{2}$, can be studied in a similar manner. We obtain that

$$
\begin{aligned}
S & =P(Y<X) \\
& =\alpha_{1} \beta_{1} \int_{0}^{\infty}\left(1+\beta_{1} x\right)^{-\left(\alpha_{1}+1\right)}\left(1+\beta_{2} x\right)^{-\alpha_{2}} d x
\end{aligned}
$$




\section{Maximum Likelihood Estimation of $S$}

Suppose that $X_{1}, X_{2}, \ldots, X_{n_{1}}$ and $Y_{1}, Y_{2}, \ldots, Y_{n_{2}}$ are two independent random samples of size $n_{1}$ and $n_{2}$ from $L\left(\alpha_{1}, \beta_{1}\right)$ and $L\left(\alpha_{2}, \beta_{2}\right)$ distributions, respectively. The log-likelihood function, with ignoring constants, is given by

$$
\begin{aligned}
\ln \mathscr{L}(\Theta \mid \mathbf{x}, \mathbf{y})= & \sum_{j=1}^{2}\left(m_{j}-r_{j}\right)\left(\ln \alpha_{j}+\ln \beta_{j}\right) \\
& +r_{1} \ln \left[1-\left(1+\beta_{1} x_{r_{1}+1}\right)^{-\alpha_{1}}\right] \\
& -\sum_{i=r_{1}+1}^{m_{1}}\left(\alpha_{1}\left(1+R_{1 i}\right)+1\right) \ln \left(1+\beta_{1} x_{i}\right) \\
& +r_{2} \ln \left[1-\left(1+\beta_{2} y_{r_{2}+1}\right)^{-\alpha_{2}}\right] \\
& -\sum_{i=r_{2}+1}^{m_{2}}\left(\alpha_{2}\left(1+R_{2 i}\right)+1\right) \ln \left(1+\beta_{2} y_{i}\right),
\end{aligned}
$$

where $\Theta=\left(\alpha_{1}, \alpha_{2}, \beta_{1}, \beta_{2}\right)$. The MLEs of $\left(\alpha_{1}, \beta_{1}\right)$, say ( $\widehat{\alpha}_{1 \mathrm{ML}}$, $\left.\widehat{\beta}_{1 \mathrm{ML}}\right)$, are obtained as the solution of the system of equations

$$
\begin{aligned}
\frac{\partial \ln \mathscr{L}}{\partial \alpha_{1}}= & \frac{m_{1}-r_{1}}{\alpha_{1}}+\frac{r_{1} \ln \left(1+\beta_{1} x_{r_{1}+1}\right)}{\left(1+\beta_{1} x_{r_{1}+1}\right)^{\alpha_{1}}-1} \\
& -\sum_{i=r_{1}+1}^{m_{1}}\left(1+R_{1 i}\right) \ln \left(1+\beta_{1} x_{i}\right)=0, \\
\frac{\partial \ln \mathscr{L}}{\partial \beta_{1}}= & \frac{m_{1}-r_{1}}{\beta_{1}}+\frac{\alpha_{1} r_{1} x_{r_{1}+1}\left(1+\beta_{1} x_{r_{1}+1}\right)^{-1}}{\left(1+\beta_{1} x_{r_{1}+1}\right)^{\alpha_{1}}-1} \\
& -\sum_{i=r_{1}+1}^{m_{1}}\left(\alpha_{1}\left(1+R_{1 i}\right)+1\right) \frac{x_{i}}{1+\beta_{1} x_{i}}=0 .
\end{aligned}
$$

In a similar way, we can obtain the MLEs of $\left(\alpha_{2}, \beta_{2}\right)$ : say $\left(\widehat{\alpha}_{2 \mathrm{ML}}, \widehat{\beta}_{2 \mathrm{ML}}\right)$.

The corresponding "ML plug-in estimation" of $S$, when $\beta_{1}=\beta_{2}=\beta$, is obtained by replacing $\alpha_{1}$ and $\alpha_{2}$ by its MLEs, $\widehat{\alpha}_{1 \mathrm{ML}}, \widehat{\alpha}_{2 \mathrm{ML}}$, and substituting them into relation (8) which yields

$$
\widehat{S}_{\mathrm{ML}}=\frac{\widehat{\alpha}_{1 \mathrm{ML}}}{\widehat{\alpha}_{1 \mathrm{ML}}+\widehat{\alpha}_{2 \mathrm{ML}}} .
$$

For the general case, $\beta_{1} \neq \beta_{2}$, the corresponding "ML plug-in estimation" of $S$ is obtained by replacing $\alpha_{1}, \beta_{1}, \alpha_{2}$, and $\beta_{2}$ by its MLEs, $\widehat{\alpha}_{1 \mathrm{ML}}, \widehat{\beta}_{1 \mathrm{ML}}, \widehat{\alpha}_{2 \mathrm{ML}}$, and $\widehat{\beta}_{2 \mathrm{ML}}$ and substituting them into relation (9) which results in obtaining $\widehat{S}_{\mathrm{ML}}$ as

$$
\begin{aligned}
\widehat{S}_{\mathrm{ML}}= & \widehat{\alpha}_{1 \mathrm{ML}} \widehat{\beta}_{1 \mathrm{ML}} \\
& \times \int_{0}^{\infty}\left(1+\widehat{\beta}_{1 \mathrm{ML}} x\right)^{-\left(\widehat{\alpha}_{1 \mathrm{ML}}+1\right)}\left(1+\widehat{\beta}_{2 \mathrm{ML}} x\right)^{-\widehat{\alpha}_{2 \mathrm{ML}}} d x .
\end{aligned}
$$

\section{Bayes Estimation}

In this section, Bayesian estimation for the probability $S$ in the stress-strength model involving Lomax distribution is obtained. The estimation is based on balanced loss function (BLF) which is introduced by Zellner [25]. We will use an extended class of BLF introduced by Jozani et al. [26]. It is of the following form:

$$
L_{\rho, \omega, \delta_{0}}^{q}(\zeta(\theta), \delta)=\omega q(\theta) \rho\left(\delta_{0}, \delta\right)+(1-\omega) q(\theta) \rho(\zeta(\theta), \delta),
$$

where $q(\cdot)$ is a suitable positive weight function and $\rho(\zeta(\theta), \delta)$ is an arbitrary loss function when estimating $\zeta(\theta)$ by $\delta$. The parameter $\delta_{0}$ is a chosen priori estimate of $\zeta(\theta)$, obtained for instance from the criterion of maximum likelihood, least squares, or unbiasedness among others. An intuitive interpretation of the BLFs is given by Ahmadi et al. [27] who argue that they give a general Bayesian connection between the case of $\omega>0$, and $\omega=0$ where $0 \leq \omega<1$. By choosing $\rho(\zeta(\theta), \delta)=(\delta-\zeta(\theta))^{2}$ and $q(\theta)=1$, the BLF is reduced to the balanced squared error loss (BSEL) function, used by Ahmadi et al. [27], in the form

$$
L_{\omega, \delta_{0}}(\zeta(\theta), \delta)=\omega\left(\delta-\delta_{0}\right)^{2}+(1-\omega) \rho(\delta-\zeta(\theta))^{2} .
$$

The corresponding Bayes estimate of the function $\zeta(\theta)$ is given by

$$
\delta_{\omega, \zeta, \delta_{0}}(\mathbf{x})=\omega \delta_{0}+(1-\omega) E(\zeta(\theta) \mid \mathbf{x}) .
$$

Also, by choosing $\rho(\zeta(\theta), \delta)=e^{a(\delta-\zeta(\theta))}-a(\delta-\zeta(\theta))-1$ and $q(\theta)=1$, we get the balanced LINEX, abbreviated as BLINEX, loss function written as

$$
\begin{aligned}
L_{a, \omega, \delta_{0}}(\zeta(\theta), \delta)= & \omega\left[e^{a\left(\delta-\delta_{0}\right)}-a\left(\delta-\delta_{0}\right)-1\right] \\
& +(1-\omega)\left[e^{a(\delta-\zeta(\theta))}-a(\delta-\zeta(\theta))-1\right] .
\end{aligned}
$$

In this case, the Bayes estimate of $\zeta(\theta)$ takes the form

$$
\delta_{a, \omega, \zeta, \delta_{0}}(\mathbf{x})=-\frac{1}{a} \ln \left[\omega e^{-a \delta_{0}}+(1-\omega) E\left(e^{-a \zeta(\theta)} \mid \mathbf{x}\right)\right],
$$

where $a \neq 0$ is the shape parameter of BLINEX loss function.

4.1. Bayes Estimation When $\beta_{1}=\beta_{2}=\beta$. Assuming that $\beta_{1}=$ $\beta_{2}=\beta$ ( $\beta$ is known) and $\alpha_{1}, \alpha_{2}$ are random variable each having gamma prior with some parameters, we can write

$$
\pi\left(\alpha_{j}\right)=\frac{\delta_{j}^{v_{j}}}{\Gamma\left(v_{j}\right)} \alpha_{j}^{v_{j}-1} e^{-\delta_{j} \alpha_{j}}, \quad \alpha_{j}>0,\left(v_{j}, \delta_{j}>0\right), j=1,2 .
$$

Since $\alpha_{j}, j=1,2$ are independent, by combining the likelihood function with the priors pdf, the joint posterior density function of $\alpha_{1}$ and $\alpha_{2}$ is given by

$$
\pi^{*}\left(\alpha_{1}, \alpha_{2} \mid \mathbf{x}, \mathbf{y}\right)=J \prod_{j=1}^{2} \alpha_{j}^{A_{j}^{*}-1} e^{-\delta_{j} \alpha_{j}-u_{j}^{*}} \sum_{s=0}^{r_{j}} C_{r_{j}, s} s^{-\alpha_{j} T_{j s}^{*}},
$$


where $A_{j}^{*}=m_{j}-r_{j}+v_{j}, T_{1 s}^{*}=T_{s}(\mathbf{x} ; \beta), T_{2 s}^{*}=T_{s}(\mathbf{y} ; \beta), u_{1}^{*}=$ $u(\mathbf{x} ; \beta), u_{2}^{*}=u(\mathbf{y} ; \beta)$, and

$$
J^{-1}=\prod_{j=1}^{2} \int_{0}^{\infty} \alpha_{j}^{A_{j}^{*}-1} e^{-\delta_{j} \alpha_{j}-u_{j}^{*}} \sum_{s=0}^{r_{j}} C_{r_{j}, s} e^{-\alpha_{j} T_{j s}^{*}} d \alpha_{j}
$$

Under the BSEL function and using (12) and (20), the proposed "Bayesian estimators" of $S$ are actually Bayesian "plugin" estimators as they are obtained by replacing $\left(\alpha_{1}, \beta_{1}, \alpha_{2}, \beta_{2}\right)$ with its Bayesian estimator $\left(\widehat{\alpha}_{1}, \widehat{\beta}_{1}, \widehat{\alpha}_{2}, \widehat{\beta}_{2}\right)$

$$
\widehat{S}_{\mathrm{BS}}=\omega \widehat{S}_{\mathrm{ML}}+(1-\omega) \int_{0}^{\infty} \int_{0}^{\infty} S \pi^{*}\left(\alpha_{1}, \alpha_{2} \mid \mathbf{x}, \mathbf{y}\right) d \alpha_{1} d \alpha_{2} .
$$

With the same argument, we can obtain Bayes estimator under the BLINEX loss function. It is obtained as follows:

$$
\begin{aligned}
\widehat{S}_{\mathrm{BL}}=-\frac{1}{a} \ln \left[\omega e^{-a \widehat{S}_{\mathrm{ML}}}+(1-\omega)\right. \\
\left.\quad \times \int_{0}^{\infty} \int_{0}^{\infty} e^{-a S} \pi^{*}\left(\alpha_{1}, \alpha_{2} \mid \mathbf{x}, \mathbf{y}\right) d \alpha_{1} d \alpha_{2}\right],
\end{aligned}
$$

where $\widehat{S}_{\mathrm{ML}}$ is the ML “plug-in” estimate of $S$ as given by (12).

4.2. Bayes Estimation when $\beta_{1} \neq \beta_{2}$. We assume that $\alpha_{j}$ and $\beta_{j}, j=1,2$, are random variables each having gamma prior with some parameters; that is,

$$
\begin{array}{r}
\pi\left(\alpha_{j} \mid \beta_{j}\right)=\frac{b_{j}^{a_{j}}}{\Gamma\left(a_{j}\right)} \alpha_{j}^{a_{j}-1} e^{-b_{j} \alpha_{j}}, \\
\alpha_{j}>0, \quad\left(a_{j}, b_{j}>0\right), \quad j=1,2, \\
\pi\left(\beta_{j}\right)=\frac{d_{j}^{c j}}{\Gamma\left(c_{j}\right)} \beta^{c j-1} e^{-d_{j} \beta_{j}}, \\
\beta_{j}>0, \quad\left(c_{j}, d_{j}>0\right), \quad j=1,2 .
\end{array}
$$

Since $\alpha_{j}$ and $\beta_{j}$ are independent, then the joint density function of $\left(\alpha_{j}, \beta_{j}\right)$ is given by

$$
\pi\left(\alpha_{j}, \beta_{j}\right)=\frac{b_{j}^{a_{j}} d_{j}^{c_{j}}}{\Gamma\left(a_{j}\right) \Gamma\left(c_{j}\right)} \alpha_{j}^{a_{j}-1} \beta_{j}^{c_{j}-1} e^{-b_{j} \alpha_{j}-d_{j} \beta_{j}}, \quad j=1,2 .
$$

Combining the likelihood function with the priors pdf yields the posterior density function of all parameters $\Theta=\left(\alpha_{1}, \alpha_{2}\right.$, $\beta_{1}, \beta_{2}$ ) as follows:

$$
\begin{aligned}
\pi^{*}(\Theta \mid \mathbf{x}, \mathbf{y})= & K \prod_{j=1}^{2} \alpha_{j}^{A_{j}-1} \beta_{j}^{B_{j}-1} e^{-u_{j}-d_{j} \beta_{j}} \\
& \times \sum_{s=0}^{r_{j}} C_{r_{j}, s} e^{-\alpha_{j}\left(T_{j s}+b_{j}\right)}
\end{aligned}
$$

where $A_{j}=m_{j}-r_{j}+a_{j}, B_{j}=m_{j}-r_{j}+c_{j}, T_{1 s}=T_{s}\left(\mathbf{x} ; \beta_{1}\right)$, $T_{2 s}=T_{s}\left(\mathbf{y} ; \beta_{2}\right), u_{1}=u\left(\mathbf{x} ; \beta_{1}\right), u_{2}=u\left(\mathbf{y} ; \beta_{2}\right)$, and

$$
\begin{aligned}
K^{-1}= & \prod_{j=1}^{2} \int_{0}^{\infty} \int_{0}^{\infty} \alpha_{j}^{A_{j}-1} \beta_{j}^{B_{j}-1} e^{-u_{j}-d_{j} \beta_{j}} \\
& \times \sum_{s=0}^{r_{j}} C_{r j, s} e^{-\alpha_{j}\left(T_{j s}+b_{j}\right)} d \alpha_{j} d \beta_{j} .
\end{aligned}
$$

Under the BSEL function, and by using (13) and (26), the Bayesian "plug-in" estimate of $S$ is given by

$$
\widehat{S}_{\mathrm{BS}}=\omega \widehat{S}_{\mathrm{ML}}+(1-\omega) \int_{\Theta} S \pi^{*}(\Theta \mid \mathbf{x}, \mathbf{y}) d \Theta .
$$

Also, based on the BLINEX loss function, the Bayes estimate of $S$ is obtained by using (13) and is written as

$$
\widehat{S}_{\mathrm{BL}}=-\frac{1}{a} \ln \left[\omega e^{-a \widehat{S}_{\mathrm{ML}}}+(1-\omega) \int_{\Theta} e^{-a S} \pi^{*}(\Theta \mid \mathbf{x}, \mathbf{y}) d \Theta\right],
$$

where $\widehat{S}_{\mathrm{ML}}$ is the ML "plug-in" estimate of $S$ as given by (13).

It may be noted, from (22), (23), (28), and (29), that the Bayes estimates of $S$ contain integrals that cannot be obtained in simple closed form, and numerical techniques must be used for computations. We, therefore, propose to consider MCMC methods.

\section{MCMC Algorithm for Bayesian Estimation}

The MCMC algorithm is conducted to compare the Bayes estimates of $S$. We consider the Metropolis-Hastings algorithm to generate samples from the conditional posterior distributions, and then we compute the Bayes estimates. For more details about the MCMC methods, see, for example, Robert and Casella [28], Upadhyaya and Gupta [29], and Jaheen and Al Harbi [30]. The Metropolis-Hastings algorithm generates samples from an arbitrary proposal distribution.

5.1. The Case When $\beta_{1}=\beta_{2}=\beta$. The conditional posteriors distributions of the parameters $\alpha_{j}, j=1,2$, can be computed and written, respectively, as

$$
\begin{gathered}
\pi^{*}\left(\alpha_{1} \alpha_{2}, \mathbf{x}, \mathbf{y}\right) \propto J \alpha_{1}^{A_{1}^{*}-1} e^{-\delta_{1} \alpha_{1}} \sum_{s=0}^{r_{1}} C_{r_{1}, s} e^{-\alpha_{1} T_{1 s}^{*}}, \\
\pi^{*}\left(\alpha_{2} \mid \alpha_{1}, \mathbf{x}, \mathbf{y}\right) \propto J \alpha_{2}^{A_{2}^{*}-1} e^{-\delta_{2} \alpha_{2}} \sum_{s=0}^{r_{2}} C_{r_{2}, s} e^{-\alpha_{2} T_{2 s}^{*}} .
\end{gathered}
$$


5.2. The Case When $\beta_{1} \neq \beta_{2}$. The conditional posteriors distributions of the parameters $\alpha_{j}$ and $\beta_{j}, j=1,2$, can be computed and written, respectively, by

$$
\begin{gathered}
\pi^{*}\left(\alpha_{1} \Theta-\alpha_{1}, \mathbf{x}, \mathbf{y}\right) \propto \alpha_{1}^{A_{1}-1} \sum_{s=0}^{r_{1}} C_{r_{1}, s} e^{-\alpha_{1}\left(T_{1 s}+b_{1}\right)}, \\
\pi^{*}\left(\beta_{1} \Theta-\beta_{1}, \mathbf{x}, \mathbf{y}\right) \propto \beta_{1}^{B_{1}-1} e^{-u_{1}-d_{1} \beta_{1}} \sum_{s=0}^{r_{1}} C_{r_{1}, s} e^{-\alpha_{1}\left(T_{1 s}+b_{1}\right)}, \\
\pi^{*}\left(\alpha_{2} \Theta-\alpha_{2}, \mathbf{x}, \mathbf{y}\right) \propto \alpha_{2}^{A_{2}-1} \sum_{s=0}^{r_{2}} C_{r_{2}, s} e^{-\alpha_{2}\left(T_{2 s}+b_{2}\right)}, \\
\pi^{*}\left(\beta_{2} \mid \Theta-\beta_{2}, \mathbf{x}, \mathbf{y}\right) \propto \beta_{2}^{B_{2}-1} e^{-u_{2}-d_{2} \beta_{2}} \sum_{s=0}^{r_{2}} C_{r_{2}, s} e^{-\alpha_{2}\left(T_{2 s}+b_{2}\right)} .
\end{gathered}
$$

The following MCMC procedure is proposed to compute Bayes estimators for $S=P(Y<X)$.

Step 1. Start with initial guess of $\alpha_{j}$ and $\beta_{j}$; say $\alpha_{j} 0$ and $\beta_{j} 0, j=1,2$.

Step 2. Set $i=1$.

Step 3. Generate $\alpha_{1 i}$ from $\pi^{*}\left(\alpha_{1} \mid \Theta-\alpha_{1}, \mathbf{x}, \mathbf{y}\right)$ and $\beta_{1 i}$ from $\pi^{*}\left(\beta_{1} \mid \Theta-\beta_{1}, \mathbf{x}, \mathbf{y}\right)$.

Step 4. Generate $\alpha_{2 i}$ from $\pi^{*}\left(\alpha_{2} \mid \Theta-\alpha_{2}, \mathbf{x}, \mathbf{y}\right)$ and $\beta_{2 i}$ from $\pi^{*}\left(\beta_{2} \mid \Theta-\beta_{2}, \mathbf{x}, \mathbf{y}\right)$.

Step 5. Compute $S\left(\alpha_{i}, \beta_{i}\right)=\alpha_{1 i} \beta_{1 i} \int_{0}^{\infty}\left(1+\beta_{1 i} x\right)^{-\left(\alpha_{1 i}+1\right)}(1+$ $\left.\beta_{2 i} x\right)^{-\alpha_{2 i}} d x$.

Step 6. Repeat Steps 2-5, $N$ times.

Now, the approximate means of $S\left(\alpha_{i}, \beta_{i}\right)$ with respect to the posterior distribution are given, respectively, by

$$
\begin{gathered}
E(S \mid \mathbf{x}, \mathbf{y})=\frac{1}{N-M} \sum_{i=M+1}^{N} S\left(\alpha_{i}, \beta_{i}\right), \\
E[\exp (-a S) \mid \mathbf{x}, \mathbf{y}]=\frac{1}{N-M} \sum_{i=M+1}^{N} \exp \left(-a S\left(\alpha_{i}, \beta_{i}\right)\right),
\end{gathered}
$$

where $M$ is the burn-in period. Therefore, the Bayes estimators of $S=S\left(\alpha_{i}, \beta_{i}\right)$ based on BSEL and BLINEX loss functions are given, respectively, by

$$
\begin{gathered}
\widehat{S}_{\mathrm{BS}}=\omega \widehat{S}_{\mathrm{ML}}+(1-\omega) E(S \mathbf{x}, \mathbf{y}) \\
\widehat{S}_{\mathrm{BL}}=-\frac{1}{a} \ln \left[\omega e^{-a \widehat{S}_{\mathrm{ML}}}+(1-\omega) E[\exp (-a S) \mid \mathbf{x}, \mathbf{y}]\right] .
\end{gathered}
$$

\section{Simulation Study}

In order to find the Bayes and likelihood estimates of the parameter $S$, a Monte Carlo study is performed following the

\begin{tabular}{|c|c|c|c|c|c|c|}
\hline \multirow{3}{*}{ CS (1) } & \multirow{3}{*}{ CS (2) } & ML & \multicolumn{4}{|c|}{ Bayes (MCMC) } \\
\hline & & \multirow{2}{*}{$S_{\mathrm{ML}}$} & \multirow{2}{*}{$S_{\mathrm{BS}}$} & \multicolumn{3}{|c|}{$S_{\mathrm{BL}}$} \\
\hline & & & & $a=-2$ & $a=1$ & $a=3$ \\
\hline \multirow{2}{*}{ (i) } & & 0.4871 & & 0.4871 & 0.4865 & 0.4860 \\
\hline & & $(0.0017)$ & $(0.00$ & $(0.0016)$ & $(0.0016)$ & $(0.0015)$ \\
\hline \multirow{2}{*}{ (iii) } & \multirow{2}{*}{ (iv) } & 0.5471 & 0.53 & 0.5393 & 0.5387 & 0.5383 \\
\hline & & $(0.0092)$ & $(0.0079)$ & $(0.0079)$ & $(0.0078)$ & $(0.0078)$ \\
\hline \multirow{2}{*}{ (v) } & \multirow{2}{*}{ (vi) } & 0.5408 & 0.5386 & 0.5388 & 0.5384 & 0.5382 \\
\hline & & $(0.0080)$ & $(0.0079)$ & $(0.0079)$ & $(0.0078)$ & $(0.0078)$ \\
\hline \multirow{2}{*}{ (vii) } & \multirow{2}{*}{ (v111) } & 0.5548 & 0.5436 & 0.5440 & 0.5434 & 0.5430 \\
\hline & & $(0.0107)$ & $(0.0088)$ & $(0.0089)$ & $(0.0088)$ & $(0.0087)$ \\
\hline
\end{tabular}
algorithms as follows.

(1) For particular values of $\alpha_{j}$ and $\beta_{j}, j=1,2$, Lomax observations of various sizes are generated for different general progressive censored schemes.
TABLE 1: Censoring schemes used in the simulation study.

\begin{tabular}{lllll}
\hline CS & $n$ & $m$ & $r$ & $R$ \\
\hline (i) & 50 & 35 & 5 & $\left(6^{*} 0,5,4^{*} 0,2,3^{*} 0,3,13^{*} 0,5\right)$ \\
(ii) & 30 & 20 & 2 & $\left(6^{*} 0,5,10^{*} 0,5\right)$ \\
(iii) & 25 & 20 & 2 & $\left(8^{*} 0,5,9^{*} 0\right)$ \\
(iv) & 35 & 30 & 5 & $\left(5^{*} 0,2,3^{*} 0,3,15^{*} 0\right)$ \\
(v) & 40 & 35 & 3 & $\left(12^{*} 0,5,19^{*} 0\right)$ \\
(vi) & 30 & 25 & 2 & $\left(8^{*} 0,3,1,1,12^{*} 0\right)$ \\
(vii) & 80 & 75 & 5 & $\left(30^{*} 0,1,10^{*} 0,2,5^{*} 0,2,22^{*} 0\right)$ \\
(viii) & 70 & 60 & 3 & $\left(2^{*} 0,2,15^{*} 0,3,10^{*} 0,3,21^{*} 0,2,5^{*} 0\right)$ \\
\hline
\end{tabular}

TABLE 2: The simulation results and estimates of $S$ when $\beta_{1}=\beta_{2}=\beta$.

(2) The ML estimates of $\alpha_{j}$ and $\beta_{j}, j=1,2$, are computed from the ML equations. The ML estimate of $S$ is computed from (13) after replacing $\alpha_{j}$ and $\beta_{j}, j=1,2$, by their ML estimates.

(3) For $N=20000, M=2000$, the Bayes estimates of $S$ are computed from (22), (23), (28), and (29) for BSEL and BLINEX loss functions based on MCMC algorithm.

(4) The squared deviations $\left(S^{*}-S\right)^{2}$ are calculated for different sample sizes and different schemes, where $S^{*}$ is ML or Bayes estimates of $S$.

(5) The above steps are repeated 1000 times, and the estimated risk (ER) is computed by averaging the squared deviations over the 1000 repetitions.

The computational results are displayed in Tables 1, 2, and 3. Table 1 shows different censoring schemes used in the simulation study. In the case of $\beta_{1}=\beta_{2}=\beta$, we take $\nu_{1}=4$, $\delta_{1}=3, \nu_{2}=3, \delta_{2}=5, \omega=0.5, \alpha_{1}=1.1339, \beta=3, \alpha_{2}=$ 0.7216 , and the true value of $S=0.4516$. For this case, Table 2 presents simulation results and the MLE, Bayes estimate, and the corresponding mean squared error is reported within bracket. In the general case, we take $a_{1}=4, b_{1}=3, a_{2}=3$, $b_{2}=2, \omega=0.5, \alpha_{1}=1.5788, \beta_{1}=1.6899, \alpha_{2}=1.5690$, $\beta_{2}=2.2002$, and the true value of $S=0.6111$. The obtained simulation results for this case are shown in Table 3. 
TABLE 3: The simulation results and estimates of $S$ when $\beta_{1} \neq \beta_{2}$.

\begin{tabular}{lcccccc}
\hline & & \multicolumn{2}{c}{ ML } & \multicolumn{5}{c}{ Bayes (MCMC) } \\
CS (1) & CS (2) & & \multicolumn{3}{c}{$S_{\mathrm{BL}}$} \\
& & $S_{\mathrm{ML}}$ & $S_{\mathrm{BS}}$ & $a=-2$ & $a=1$ & $a=3$ \\
\hline \multirow{2}{*}{ (i) } & (ii) & 0.7217 & 0.6968 & 0.6977 & 0.6964 & 0.6954 \\
& & $(0.0124)$ & $(0.0077)$ & $(0.0078)$ & $(0.0076)$ & $(0.0074)$ \\
(iii) & (iv) & 0.6440 & 0.6085 & 0.6102 & 0.6077 & 0.6059 \\
& & $(0.0013)$ & $(0.0004)$ & $(0.0004)$ & $(0.0004)$ & $(0.0005)$ \\
(v) & (vi) & 0.7001 & 0.6731 & 0.6741 & 0.6726 & 0.6716 \\
& & $(0.0080)$ & $(0.0041)$ & $(0.0042)$ & $(0.0040)$ & $(0.0039)$ \\
(vii) & (viii) & 0.6771 & 0.6418 & 0.6434 & 0.6410 & 0.6395 \\
& & $(0.0044)$ & $(0.0010)$ & $(0.0011)$ & $(0.0010)$ & $(0.0009)$ \\
\hline
\end{tabular}

\section{Conclusions}

In this paper, the estimation of the stress-strength parameter, $S$, for two Lomax distributions under general progressive type-II censoring has been considered. The maximum likelihood and Bayes estimators of the stress-strength parameter have been derived. The MCMC method is used for computing Bayes estimates. It is observed that Bayes estimators outperform the ML estimators in small samples, while the estimators are almost equally efficient in large samples. It may be noted, from Tables 2 and 3, that the Bayes estimates have the smallest mean squared errors as compared with their corresponding maximum likelihood estimates. Based on the obtained results in this study and because of the need to deal with small samples in life testing, we recommend to use Bayes estimators in place of ML estimators.

\section{Acknowledgments}

The authors are grateful to the editor for his valuable comments and suggestions which improved the presentation of the paper. This Project was funded by the Deanship of Scientific Research (DSR), King Abdulaziz University, Jeddah, under Grant no. 268/130/1432. The authors, therefore, acknowledge with thanks DSR support for Scientific Research.

\section{References}

[1] A. Balkema and L. de Haan, "Residual life time at great age," Annals of Probability, vol. 2, no. 5, pp. 792-804, 1974.

[2] M. C. Bryson, "Heavy-tailed distributions: properties and tests," Technometrics, vol. 16, no. 1, pp. 61-68, 1974.

[3] M. Ahsanullah, "Record values of Lomax distribution," Statistica Nederlandica, vol. 41, no. 1, pp. 21-29, 1991.

[4] N. Balakrishnan and M. Ahsanullah, "Relations for single and product moments of record values from Lomax distribution," Sankhya B, vol. 56, no. 2, pp. 140-146, 1994.

[5] A. Childs, N. Balakrishnan, and M. Moshref, "Order statistics from non-identical right-truncated Lomax random variables with applications," Statistical Papers, vol. 42, no. 2, pp. 187-206, 2001.
[6] B. C. Arnold, E. Castillo, and J. M. Sarabia, "Bayesian analysis for classical distributions using conditionally specified priors," Sankhya B, vol. 60, no. 2, pp. 228-245, 1998.

[7] M. M. El-Din, H. M. Okasha, and B. Al-Zahrani, "Empirical bayes estimators of reliability performances using progressive type-II censoring from Lomax model," Journal of Advanced Research in Applied Mathematics, vol. 5, no. 1, pp. 74-83, 2013.

[8] H. A. Howlader and A. M. Hossain, "Bayesian survival estimation of Pareto distribution of the second kind based on failurecensored data," Computational Statistics and Data Analysis, vol. 38, no. 3, pp. 301-314, 2002.

[9] M. E. Ghitany, F. A. Al-Awadhi, and L. A. Alkhalfan, "MarshallOlkin extended lomax distribution and its application to censored data," Communications in Statistics, vol. 36, no. 10, pp. 1855-1866, 2007.

[10] E. Cramer and A. B. Schmiedt, "Progressively type-II censored competing risks data from Lomax distributions," Computational Statistics and Data Analysis, vol. 55, no. 3, pp. 1285-1303, 2011.

[11] N. Johnson, S. Kotz, and N. Balakrishnan, Continuous Univariate Distribution, vol. 1, John Wiley \& Sons, New York, NY, USA, 2nd edition, 1994.

[12] B. C. Arnold, Pareto Distributions, International Cooperative, Silver Spring, Md, USA, 1983.

[13] S. Kotz, Y. Lumelskii, and M. Pensky, The Stress-Strength Model and Its Generalizations: Theory and Applications, World Scientific, New York, NY, USA, 2003.

[14] A. Baklizi, "Likelihood and Bayesian estimation of $\operatorname{Pr}(X<$ $Y$ ) using lower record values from the generalized exponential distribution," Computational Statistics and Data Analysis, vol. 52, no. 7, pp. 3468-3473, 2008.

[15] M. Z. Raqab, M. T. Madi, and D. Kundu, "Estimation of $P(Y<X)$ for the three-parameter generalized exponential distribution," Communications in Statistics, vol. 37, no. 18, pp. 2854-2864, 2008.

[16] D. Kundu and M. Z. Raqab, "Estimation of $R=P(Y<X)$ for three-parameter Weibull distribution," Statistics and Probability Letters, vol. 79, no. 17, pp. 1839-1846, 2009.

[17] H. Panahi and S. Asadi, "Inference of stress-strength model for a lomax distribution," Proceedings of World Academy of Science, Engineering and Technology, vol. 7, no. 55, pp. 275-278, 2011.

[18] A. A. Soliman, A. Y. Al-Hossain, and M. M. Al-Harbi, "Predicting observables from Weibull model based on general progressive censored data with asymmetric loss," Statistical Methodology, vol. 8, no. 5, pp. 451-461, 2011.

[19] N. Balakrishnan and R. A. Sandhu, "A simple simulational algorithm for generating progressive type-II censored samples," The American Statistician, vol. 49, no. 2, pp. 229-230, 1995.

[20] B. Saraoglu, I. Kinaci, and D. Kundu, "On estimation of $R=$ $P(Y<X)$ for exponential distribution under progressive typeII censoring," Journal of Statistical Computation and Simulation, vol. 82, no. 5, pp. 729-744, 2012.

[21] N. Balakrishnan and R. Aggarwala, Progressive Censoring: Theory, Methods and Applications, Birkhauser, Boston, Mass, USA, 2000.

[22] B. Nandi and A. B. Aich, "A note on confidence bounds for $P(Y$ $>X$ ) in bivariate normal samples," Sankhya B, vol. 56, no. 2, pp. 129-136, 1994.

[23] A. Barbiero, "Interval estimators for reliability: the bivariate normal case," Journal of Applied Statistics, vol. 39, no. 3, pp. 501512, 2012. 
[24] F. J. Rubio and M. F. Steel, "Bayesian inference for $P(X<Y)$ using asymmetric dependent distributions," Bayesian Analysis, vol. 7, no. 3, pp. 771-792, 2012.

[25] A. Zellner, "Bayesian and non-Bayesian estimation using balanced loss functions," in Statistical Decision Theory and Methods, J. O. Berger and S. S. Gupta, Eds., pp. 337-390, Springer, New York, NY, USA, 1994.

[26] M. J. Jozani, E. Marchand, and A. Parsian, "On estimation with weighted balanced-type loss function," Statistics and Probability Letters, vol. 76, no. 8, pp. 773-780, 2006.

[27] J. Ahmadi, M. J. Jozani, E. Marchand, and A. Parsian, "Bayes estimation based on $k$-record data from a general class of distributions under balanced type loss functions," Journal of Statistical Planning and Inference, vol. 139, no. 3, pp. 1180-1189, 2009.

[28] C. P. Robert and G. Casella, Monte Carlo Statistical Methods, Springer, New York, NY, USA, 2005

[29] S. K. Upadhyay and A. Gupta, "A bayes analysis of modified Weibull distribution via Markov chain Monte Carlo simulation," Journal of Statistical Computation and Simulation, vol. 80, no. 3, pp. 241-254, 2010.

[30] Z. F. Jaheen and M. M. Al Harbi, "Bayesian estimation for the exponentiated Weibull model via Markov chain Monte Carlo simulation," Communications in Statistics: Simulation and Computation, vol. 40, no. 4, pp. 532-543, 2011. 

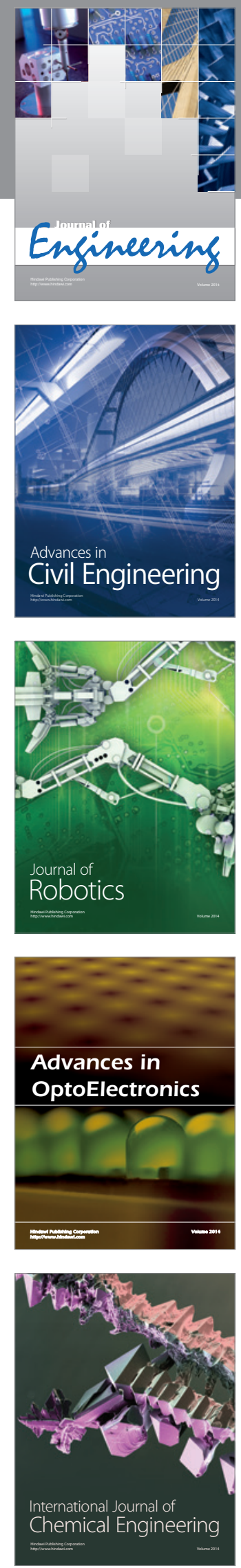

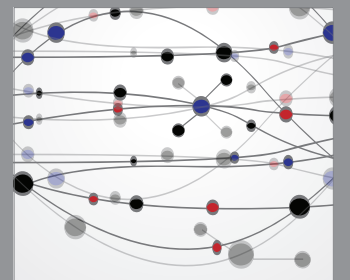

The Scientific World Journal
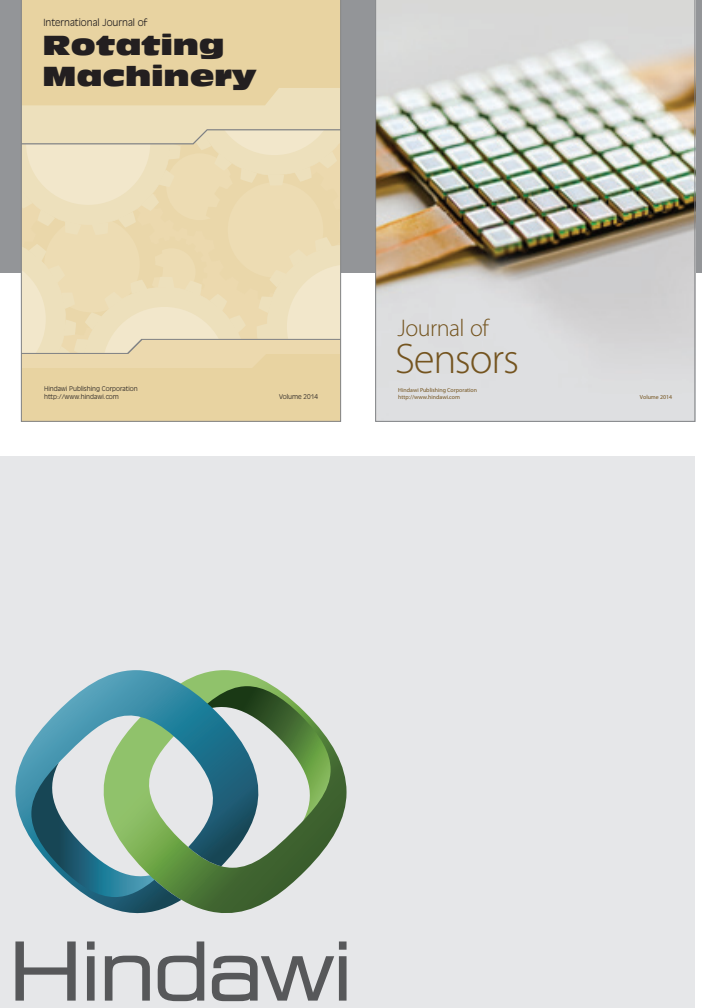

Submit your manuscripts at http://www.hindawi.com
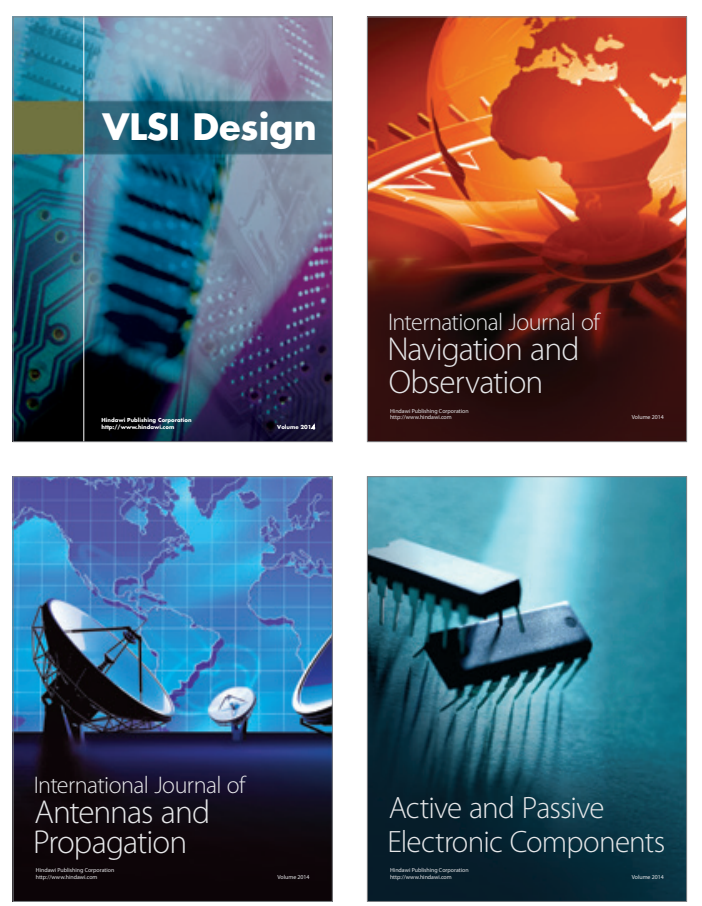
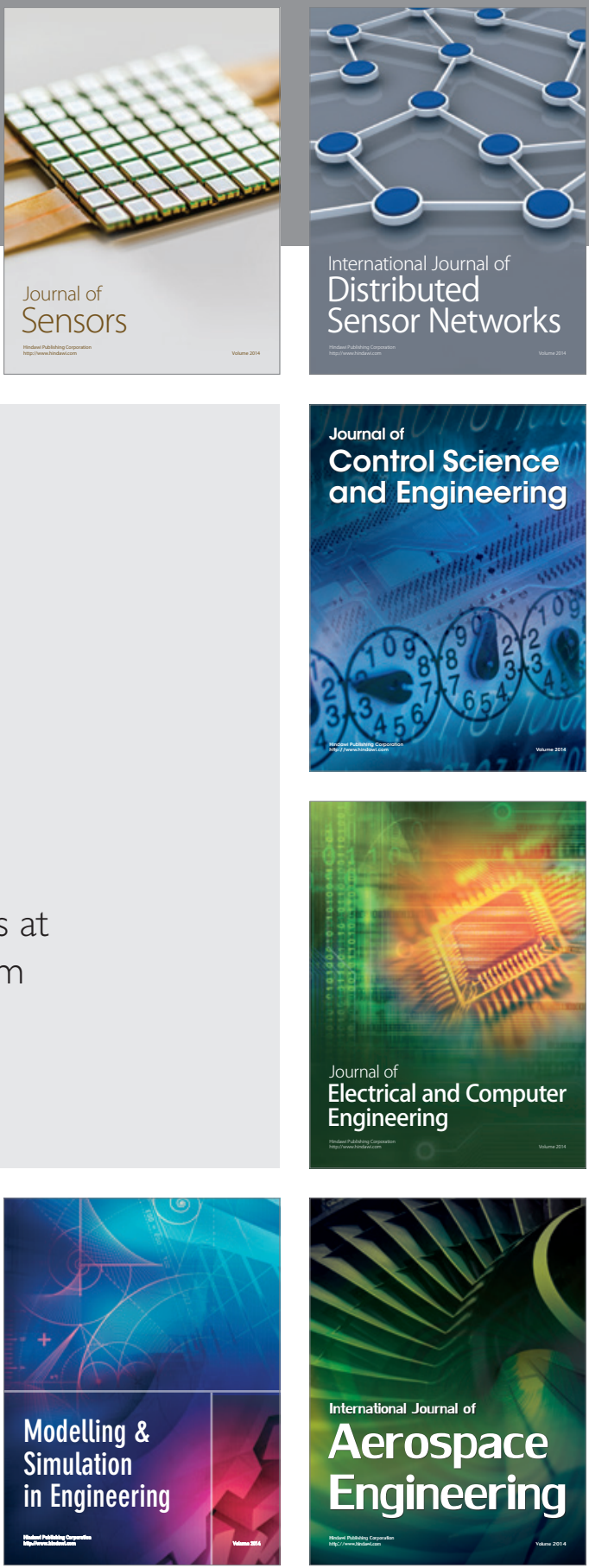

Journal of

Control Science

and Engineering
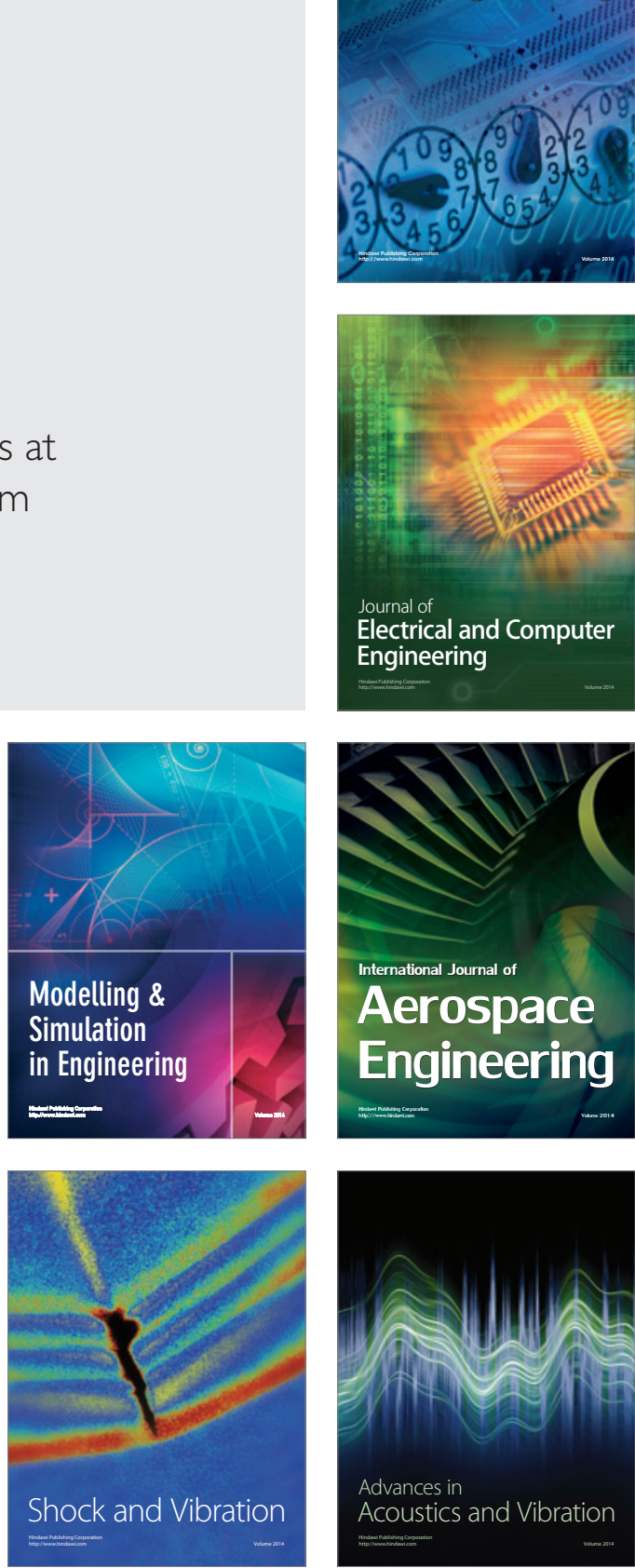